YI SHEN, Ph.D. ${ }^{1,2}$

E-mail: shen_yi1979@njau.edu.cn

GANG REN, Ph.D. ${ }^{1}$

(Corresponding author)

E-mail: rengang@seu.edu.cn

YANG LIU, M.A. ${ }^{1,2}$

E-mail: 774841985@qq.com

${ }^{1}$ School of Transportation, Southeast University

Nanjing 210096, China

2 College of Information Science and Technology

Nanjing Agricultural University, Nanjing 210095, China
Science in Traffic and Transport

Preliminary Communication

Submitted: 12 Oct. 2016

Accepted: 10 Oct. 2017

\title{
TIMETABLE DESIGN FOR MINIMIZING PASSENGER TRAVEL TIME AND CONGESTION FOR A SINGLE METRO LINE
}

\begin{abstract}
This paper brings a proposal for a timetable optimization model for minimizing the passenger travel time and congestion for a single metro line under time-dependent demand. The model is an integer-programming model that systemically considers the passenger travel time, the capacity of trains, and the capacity of platforms. A multi-objective function and a recursive optimization method are presented to solve the optimization problem. Using the model we can obtain an efficient timetable with minimal passenger travel time and minimal number of congestion events on platforms. Moreover, by increasing the number of dispatches, the critical point from congestion state to free-flow state and the optimal timetable with minimal cost for avoiding congestion on platforms can be obtained. The effectiveness of the model is evaluated by a real example. A half-regular timetable with fixed headways in each operation period and an irregular timetable with unfixed headway are investigated for comparison.
\end{abstract}

\section{KEY WORDS}

metro line; timetable optimization; time-dependent demand; congestion;

\section{INTRODUCTION}

As an important public transit system, the metro networks have many advantages for passengers such as large capacity, high speed, and high reliability. The improvement of the service of the metro networks, especially the reduction of passenger waiting times, has attracted many recent efforts in cities. Some dynamic operation strategies, such as dynamically adjusting train speed or dwell time at stations [1] are proposed. However, these dynamic operation strategies usually imply complex operations and are difficult to implement in real applications. Compared with the dynamic operation strategies, the timetable methods, which are relatively stable and secure, are accepted as the most straightforward and effective solutions [2].
There are quite some contributions in literature on timetable optimization for urban rail transit lines. Without detailed passenger demand data, previous studies on scheduling mainly focused on an idealized transit and attempted to estimate passenger waiting time. Some early studies $[3,4]$ used continuum fluid-flow models to approximate the passenger loading patterns on a single origin-destination route and calculated the total passenger waiting time by various anaIytical formulas. In general, when the passenger arrival process at stations follows some particular probability distributions, such as uniform and Poisson distributions, a regular schedule with fixed headway between consecutive vehicles can reduce the total passenger waiting time effectively $[5,6]$. However, using regular schedules may result in longer passenger waiting time and travel time during over-saturated periods [7]. Some studies $[7,8]$ paid attention to semi-regular or half-regular timetables. For these timetables, a day is usually divided into several periods, such as peak periods and off-peak periods, and each period uses its own fixed headway.

In fact, the real passenger demand is time-dependent or dynamic. In recent years, efficient and robust timetables under dynamic demand have been the topic of many research papers. For reducing the passenger waiting time at the transfer stations, some researchers focus on transit coordination and synchronization among different lines by timetables. Wong et al. [9] used a mixed integer programming optimization method to minimize the transfer waiting time of passengers in Hong Kong Mass Transit Railway (MTR). Hadas and Ceder [10] introduced a synchronized timetable to reduce the waiting time caused by transfers. Wu et al. [11] proposed a timetable synchronization optimization model to reduce the passengers waiting time at transfer stations. These synchronization methods 
can be also used to solve the problem when missing a simultaneous intersection will result in long delay, especially for longer headway and transit services in rural areas $[12,13]$. For reducing the passenger waiting time at the platforms of a line, some researchers focused on searching the optimal dispatch time, defined as the optimal departure times at the start station for each train that can better meet the dynamic passenger demand and reduce the passenger waiting time. These methods are based on the assumption that dwell and running times of all trains are pre-given and constant. Albrecht [14] proposed an optimized timetable incorporated with variable passenger demand. Barrena et al. [15] considered dynamic passenger demand and presented a branch-and-cut algorithm to minimizing average passenger waiting time. Sun et al. [12] formulated three optimization models to design demand-sensitive timetables. Niu and Zhou et al. [16, 17] studied the train scheduling problems to minimize the passenger waiting time with time-dependent demand, skip-stop patterns, and over-saturated conditions.

It is noted that only focusing on minimizing passenger waiting time may lead to congestion on trains and stations, especially for peak-hour demand. The capacity of trains and stations, which is closely related to congestion, is a key problem to the station passenger flow organization, station device schemes, and train operation [18]. The definition of capacity has been regarded as a significant issue for decades in the railway industry $[19,20,21]$. The capacity of trains and stations may vary according to factors such as train speed, commercial stops, train heterogeneity, distance between railway signals, and timetable robustness [22], and has been studied from local [19] and systematic [18] points of view. Besides the capacity, it should be also pointed out that the distribution of passengers along the platforms and inside the trains is complex and heterogeneous in reality, especially for crowded situations [23]. For the platforms, the level of service may range from large space to personal space which is equivalent to the approximate area of the body ellipse. Similarly, the situation in trains may range from many vacant seats to the over-crowded case when no one can move freely [23]. Moreover, it was found that the dwell time of trains can be influenced by boarding and alighting, or total interchanging passengers [13, 23].

The research papers $[12,14,15]$ have considered the passenger waiting time and the capacity of trains under time-dependent or dynamic demand for the fact that the waiting passenger may not be able to board the next arriving train under over-saturated conditions. Zhu et al. assumed that the passenger demand is steady, and created an efficient timetable with minimal passenger cost and operation cost through considering the capacity of trains and stations [24]. However, the real passenger demand is dynamic, and some waiting passengers may not be able to board the next arriving train even if the train has a lot of free capacity when congestion on platforms occurs. Beside the passenger waiting time, minimizing the travel time of passengers, is actually more meaningful and widely used in practice. Therefore, to obtain an efficient timetable under dynamic demand, we must consider the passenger travel time which mainly includes the passenger waiting time, the dwell time of trains on stations, and the running time of trains between adjacent stations.

This paper investigates the scheduling problem of a single metro line, and proposes a timetable optimization model for minimizing the passenger travel time and congestion on platforms under time-dependent demand. The model considers the passenger travel time, the capacity of trains, and the capacity of platforms systematically. A multi-objective function and a recursive method incorporated by the genetic algorithm are presented to solve the optimization problem. We obtain a train timetable with minimum passenger travel time and a number of congestion events on platforms under the given train capacity. Moreover, by increasing the number of dispatches, the critical point from congestion state to free-flow state and the optimal timetable with minimal cost for avoiding congestion on platforms under high demand can be obtained. The model is evaluated on the data of the first stage project of Nanjing Metro line 1 in China. To make comparisons, a half-regular timetable with fixed headway in each period and an irregular timetable with unfixed headway that only considers the capacity of trains, are investigated for comparison sake.

\section{THE MODEL}

\subsection{Assumptions and parameters}

Assumption 1. We consider a single metro line, as shown in Figure 1. The stations are numbered as $1,2, \ldots, 2 M$. Geographically, the station pairs $(1,2 M),(1,2 M-1), \ldots,(M, M+1)$ correspond to two opposite directions of the same stations. This paper assumes that the passenger demands on two reverse directions, $1 \rightarrow M$ and $M+1 \rightarrow 2 M$, are independent of each other, and all boarded passengers will alight from the train at the start station $M$ and return station $2 M$.

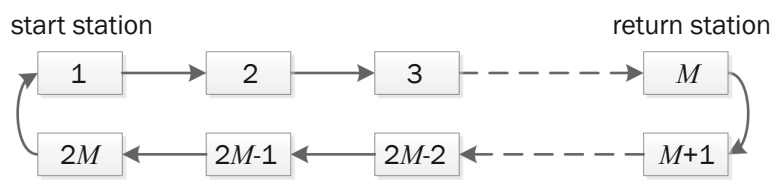

Figure 1 - A single loop metro line 
Assumption 2. In order to facilitate the problem formulation and simplification, this paper assumes that all the waiting passengers follow the First-In-First-Out(FIFO) principle to board a train, and the passengers on the arriving train alight first. Two situations, under-saturated conditions and over-saturated conditions, for an urban rail station are considered. For under-saturated conditions, all passengers waiting at a platform can always board the arriving train, and there is no left-behind passenger on the platform after the train leaves. Under over-saturated conditions, only a part of the passengers who arrive early can board the arriving train. For the boarded passengers, we assume that all of them can alight from the train at their destinations.

Assumption 3. We divide the study period equally into several extremely tiny time intervals (e.g. 0.2 second), and then refer to any particular time interval as time and use $t$ to index it. The modelling time interval length is sufficiently small, which allows only one passenger from the outside arriving at a station during a single time interval. Without this assumption, if the remaining train capacity at time $t$ is 1 , and if 2 or more passengers arrive at a station during a time interval, then none of passengers can board the train in order not to violate the capacity constraints. Furthermore, all passengers leave the station as soon as they arrive at their destinations.

The parameters used in the model are listed below. This includes the given constant inputs, model variable as well as indices.

Indices:

$u \quad$ - index of stations;

j - index of trains;

$t \quad$ - index of modelling time intervals;

Variables:

$A^{u}(t)$ - the passenger arriving rate at the platform of station $u$ (number of arriving passengers during a time interval, $A^{u}(t)=0$ or 1 );

$f_{j}^{u, v}$ - number of passengers travelling from station $u$ to station $v$ that board train $j$ at station $u$;

$I_{j}^{u} \quad$ - number of passengers boarding train $j$ at station $u$;

$O_{j}^{u} \quad$ - number of passengers alighting from train $j$ at station $u$ and leaving the station;

$E_{j}^{u} \quad$ - number of passengers entering the platform of station $u$ in an effective loading time window $t \in\left(T D_{j-1}^{u}, T D_{j}^{u}\right]$

$T A_{j}^{u}$ - arrival time of train $j$ at station $u$;

$T D_{j}^{u}$ - departure time of train $j$ from station $u$;

$P_{j}^{u} \quad$ - the latest time for passengers arriving at the platform of station $u$ to board train $j$;

$S_{j}^{u} \quad$ - number of passengers remaining on the platform of station $u$ when train $j$ reaches its capacity and departs from the station;

$T_{j}^{u} \quad$ - number of passengers on train $j$ that want to continue to a further destination after the train departs from station $u$;
$C_{n} \quad$ - number of congestion events on platforms.

A congestion event occurs and is counted if the number of waiting passengers exceeds the platform capacity of a station when a train leaves the station.

$C_{\text {min }}$ - minimum number of congestion events on platforms.

Given inputs:

tc - the capacity of a train;

$p c \quad$ - the capacity of a platform;

$K \quad$ - the total number of trains departing from the start station during the study period;

$h$ - minimum headway;

$\alpha \quad$ - minimum average passenger load rate;

$\bar{J} \quad$ - the set of trains that need to satisfy the minimum average passenger loading rate requirements.

\subsection{Constraints}

In the time range of $\left(T D_{j-1}^{u}, T D_{j}^{u}\right\rfloor$ at station $u$, the number of passengers that arrive on the platform of station $u$ is

$$
\begin{aligned}
& E_{j}^{u}=\sum_{t \in\left(T D_{j-1}^{u}, T D_{j}^{u}\right]} A^{u}(t) \\
& \text { for } u=1,2, \ldots, 2 M-1 ; j=1,2, \ldots, K
\end{aligned}
$$

The number of passengers alighting from train $j$ at station $u$ is

$$
\left\{\begin{array}{l}
O_{j}^{u}=\sum_{u^{\prime}=1}^{u-1} f_{j}^{u^{\prime}, u} \\
\text { for } u=2,3, \ldots M ; j=1,2, \ldots, K ; O_{j}^{1}=0 \\
O_{j}^{u}=\sum_{u^{\prime}=M+1}^{u-1} f_{j}^{i^{\prime}, u} \\
\text { for } u=M+2, M+3, \ldots, 2 M ; j=1,2, \ldots, K ; \\
\quad O_{j}^{M+1}=0
\end{array}\right.
$$

where $f_{j}^{u, v}=0$, if $u=\in(2,3, \ldots, M)$ and $v \in(M+1, M+2, \ldots, 2 M)$, or $u \in(M+1, M+2, \ldots, 2 M)$ and $v \in(2,3, \ldots, M)$.

In the time range of $\left(P_{j-1}^{u}, P_{j}^{u}\right]$ at station $u$, the number of passengers boarding train $j$ is

$$
\begin{aligned}
& I_{j}^{u}=\sum_{t \in\left(P_{j-1}^{u}, P_{j}^{u}\right]} A^{u}(t) \\
& \text { for } u=1,2, \ldots, 2 M-1 ; j=1,2, \ldots, K ; P_{j=0}^{u}=0
\end{aligned}
$$

In this model, the flow conservation constraints of both trains and stations are considered. According to the illustration of the train operations and passenger flow, as shown in Figure 2, the flow conservation constraint of passengers per train is represented as follows.

$$
\begin{aligned}
& T_{j}^{u}=T_{j}^{u-1}+I_{j}^{u}-O_{j}^{u} \\
& \text { for } u=2,3, \ldots, 2 M ; j=1,2, \ldots, K
\end{aligned}
$$




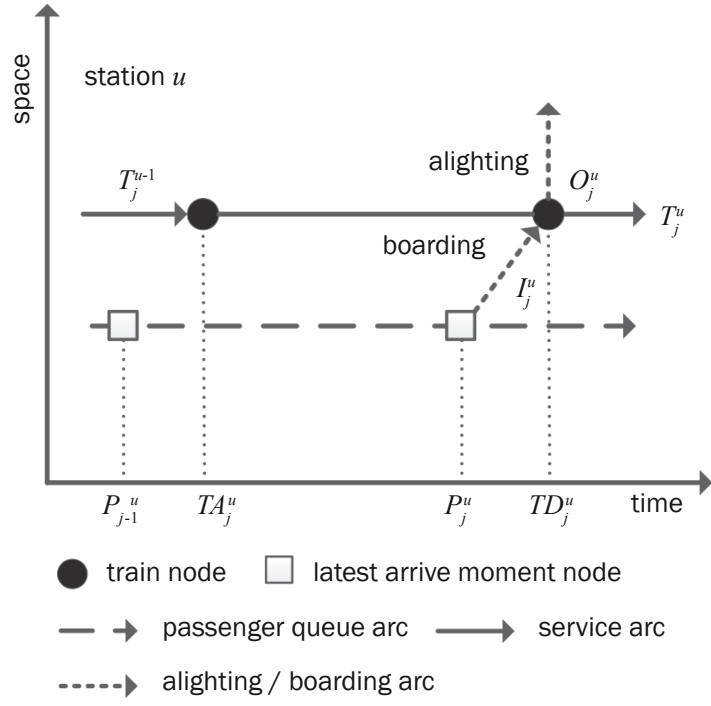

Figure 2 - Illustration of train operations and passenger flows

In Figure 2, the dwell time of train $j$ is defined as the time interval between $T A_{j}^{u}$ and $T D_{j}^{u}$. A set of nodes associated with the latest arriving moment node (corresponding to $P_{j}^{u}$ ) separates the time coordinate into several mutually exclusive intervals for each effective passenger-loading period.

Figure 3 shows the process for the passengers entering and leaving the platform of a station, and the flow conservation constraint of passengers per station can be represented as follows.

$$
\begin{aligned}
& S_{j}^{u}=S_{j-1}^{u}+E_{j}^{u}-I_{j}^{u} \\
& \text { for } u=1,2,3, \ldots, 2 M ; j=1,2,3, \ldots, K ; S_{0}^{u}=0
\end{aligned}
$$

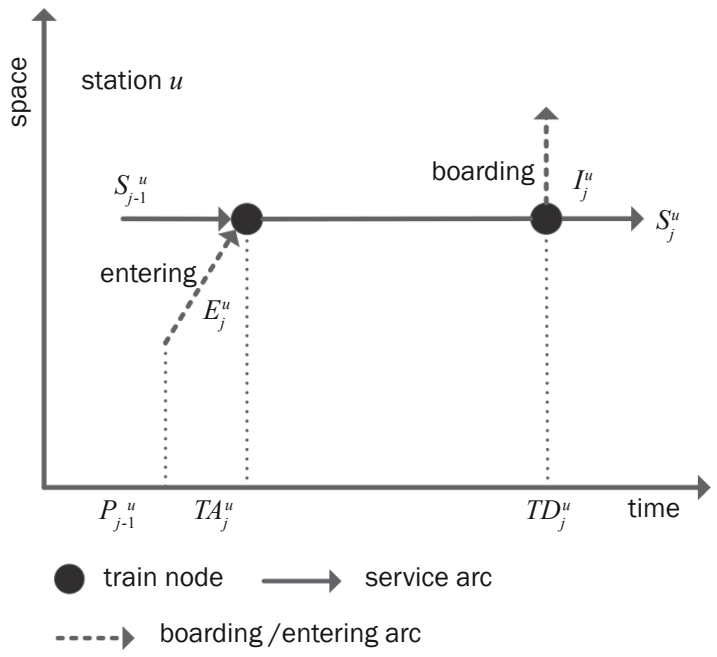

Figure 3 - Illustration of the process for passengers who enter and leave a station

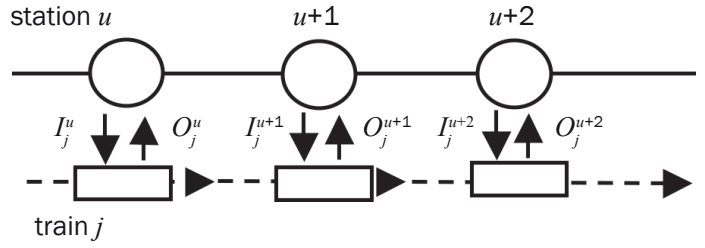

Figure 4 - The passenger flow between the trains and stations

The passenger flow between the trains and stations is shown in Figure 4. When train $j$ arrives at station $u$, it will trigger an arriving event, and the interactions between the train and the station are $I_{j}^{u}$ and $O_{j}^{u}$.

This study assumes that the passengers waiting for the last train could board the final run. Then, the supply constraint is

$S_{K-1}^{u}=E_{K}^{u} \leq t c-T_{K}^{u-1}+O_{K}^{u}$

for $u=1,2,3, \ldots, 2 M-1$

This constraint indicates that the train supply meets the total passenger demands. For train $j, T_{j}^{u} \leq t c$, but for station $u, S_{j}^{u} \leq p c$ is not always satisfied especially for peak-hour demand. Therefore, an alternative condition can be relied on, i.e. the minimal number of congestion events on platforms denoted by $C_{\text {min }}$.

For a certain train, it must arrive at the start station before we re-dispatch it. We use $T$ to represent the minimum recovery allowance time for the finished train at final destination to be ready for a new service at the start station, which is called pull-out time here. The corresponding constraint is

$T D_{j+T r a i n s \_i n \_s c h e d u l e}^{1}-T A_{j}^{2 M} \geq T$

for $j=1,2, \ldots, K$-Trains_in_schedule

In fact, train $j$ and train $j+$ Trains_in_schedule in schedule are the same train.

\subsection{Passenger loading time and requirement}

In the case of under-saturated conditions, for train $j$, at station $u$, the latest arrival time is

$P_{j}^{u}=T D_{j}^{u}$ for $j=1,2, \ldots, K ; P_{j=0}^{u}=0$

In the case of over-saturated conditions, for train $j$ at station $u$, the latest arrival time is

$P_{j}^{u}=\max \left\{\tau \mid \sum_{t \in\left(P_{j-1}^{u}, \tau\right]} A^{u}(t) \leq t c-T_{j}^{u-1}+O_{j}^{u}\right\}$

for $j=1,2, \ldots, K ; P_{j=0}^{u}=0$

The passenger loading requirement is used to trade off the benefits between the transit service provider and the transit service users, which can be quantified by the ratio of the overall number of on-board passengers to the total utilized train capacity [15]. It is reasonable to allow a few trains to have lower load rates than required during early morning or late night 
hours. We denote the set of trains that need to satisfy the minimum passenger loading rate requirements by $\bar{J}$. By limiting the loaded passengers within a reasonable range, the passenger loading requirement constraint is presented as follows:

$$
\frac{1}{(2 M-2) \cdot t c} \cdot \sum_{u=1}^{2 M} T_{j}^{u} \geq \alpha \text { for } j \in \bar{J}
$$

where $2 M-2$ is the number of segments in each service cycle of a train because the trains run empty at start station and return station.

\subsection{Multi-objective function}

At station $u$, the waiting time of a passenger is $T D_{j}^{u}-t$ before train $j$ leaves the station for passengers arriving time $t \in\left(P_{j-1}^{u}, P_{j}^{u}\right]$ Thetotal passengerwaitingtimesover the study period is $\sum_{j=1}^{K} \sum_{u=1}^{2 M} \sum_{t \in\left(P_{j-1}^{u}, P_{j}^{u}\right]} A^{u}(t) \cdot\left(T D_{j}^{u}-t\right)$. When the total travel time of passengers $Z$ is considered, the waiting time of passengers inside the trains should be added. For temporary peak-hour demand, the congested platforms may always exist. Therefore, the multi-objective function is proposed as follows.

$$
\left\{\begin{aligned}
\min Z & =\sum_{j=1}^{K} \sum_{u=1}^{2 M} \sum_{t \in\left(T D_{j-1}^{u}, P_{j}^{u}\right]} A^{u}(t) \cdot\left(T D_{j}^{u}-t\right)+ \\
& +\sum_{j=1}^{K} \sum_{u=1}^{2 M-1} \sum_{v=2}^{2 M} f_{j}^{u, v} W R_{j}^{u, v} \\
\min C_{n} &
\end{aligned}\right.
$$

where $W R_{j}^{u, v}$ is the travel time of train $j$ from station $u$ to station $v$, which includes the total dwell time $\sum_{s=u+1}^{v-1} W_{j}^{s}$ and total running time $\sum_{s=u}^{v} R_{j}^{s, s+1}$ of trains between stations $u$ and $v . W_{j}^{s}$ is the dwell time at station $s$ for train $j$ and $R_{j}^{s, s+1}$ is the running time between two adjacent stations $s$ and $s+1$ for train $j$.

The above multi-objective function has the property of non-dominated solutions. A conventional method for solving multi-objective functions is using adaptive weight approaches which set adaptive weights on different objective functions and transform them into a single-objective function. Here, since we mainly focus on $C_{n}$, inspired by the idea of preference-based optimization methods, a recursive method to solve Equation 11 is proposed. In our method, it is first assumed that there are no congested stations; thus the first ideal point of $C_{n}$ is 0 . Then, GA method is used to optimize $Z$. If the ideal point cannot be realized after a number of iterations such as 400 , i.e. there is no feasible solution that satisfies the ideal point, the ideal point is increased by step 1 and $Z$ is optimized again. Based on this process, we can finally obtain the minimized $Z$ under the condition of minimized $C_{n}$. Our method runs in a recursive way as follows.

Step 1: The minimized $C_{n}$ is set with initial value 0 .
Step 2: Use the genetic algorithm to solve the multi-objective function denoted by Equation 11. If the genetic algorithm fails to find a feasible solution that satisfies $C_{n}$, go to Step 3.

Step 3: $C_{n}=C_{n}+1$, go to Step 2 .

The above process runs until the optimal solution is obtained. In order to obtain a timetable that satisfies $C_{n}=0$, we can increase the number of dispatches which is determined by the fleet-size. In this case, the fleet-size may increase because the number of dispatches is limited under a fixed fleet-size.

\section{SOLUTION OF THE MODEL}

The integer-programming model proposed in Section 2 deals with a complex, non-linear programming problem. This problem is difficult to solve by gradient-based methods or commercial optimization solvers. We adopt the genetic algorithm which is based on natural selection to solve the optimization problem. The key variables are the departure times of trains at the start station, the dwell time for each train at each station, and the running time for each train between station pairs, which can be represented by a chromosome. In Figure 5 we plot a simple metro network with two lines to explain the application of our method. At first, we use GA method to optimize the departure time, the dwell time, and the running time for line 1. Then we can apply these optimized variables to line 1 to decrease the total passenger travel time on line 1. For the transfer station S4 on line 1, the passengers that transfer to or from line 2 can be considered to leave or enter S4 for simplicity. When for line 2, the process is the same with that of line 1.

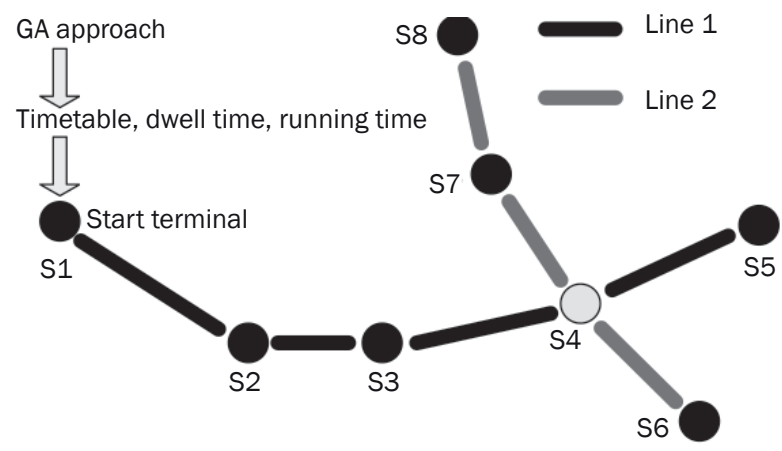

Figure 5 - Illustration of the application of our method

\subsection{Representation of a chromosome}

Each randomly generated gene corresponds to a possible time point that represents a decision. We propose a special chromosome to represent a decision of our method. The chromosome includes three parts: departure time of each train, the dwell time of the trains at each station, and the running time of the 


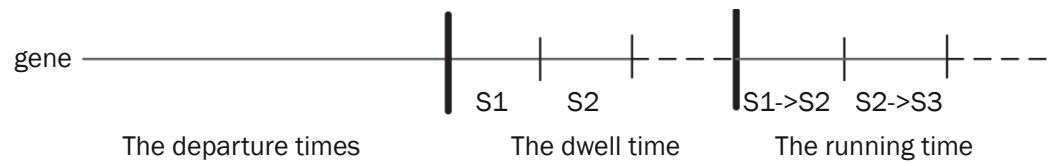

a) The overall structure of a chromosome (S1 - station 1, S2 - station 2)

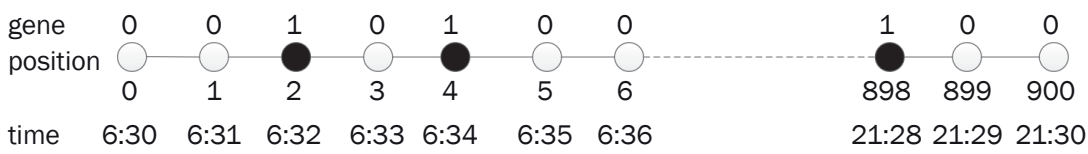

b) The departure times of trains at the start station

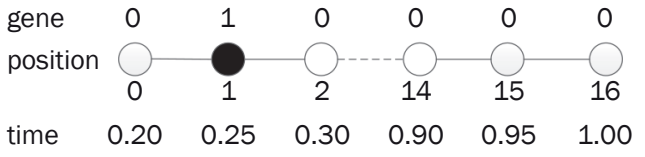

c) The dwell time at a station

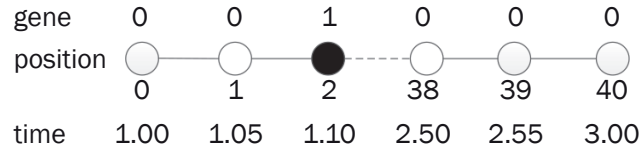

d) The running time of the trains between two adjacent stations

Figure 6 - Illustration of the three parts of the chromosome

trains between each station pair. For the departure time, the binary string ' 1 ' indicates a departure and ' 0 ' indicates no-departure at the corresponding moment. For the dwell time and running time, the binary string ' 1 ' means their corresponding value. The interval of the binary string is a scale problem. Here, the interval of the departure time of each train is 1 minute, and the interval of dwell time and running time is $0.05 \mathrm{~min}$. In Figure 6, an example of the chromosome is plotted. As the example shows, the departure times of trains at the start station are 6:32, 6:34, 21:28, etc. The dwell time at a station is $0.25 \mathrm{~min}$. and the running time between a station pair is $1.10 \mathrm{~min}$. For high resolution, the density of chromosome points needs to be increased and the time scale modified.

\subsection{Genetic operators}

As shown in Figure 6a, the chromosome includes three parts, and the crossover and mutation process for different parts are executed in their own domains. For the departure time, our custom designed crossover process works as follows.

Step 1: Two points are selected at random as the cross_start and cross_end respectively. The segment between the cross_start and cross_end is selected as a candidate for cross operation, as shown in Figure 7.

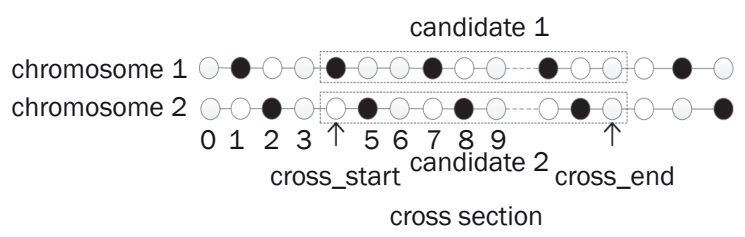

Figure 7 - A randomly selected segment in a randomly selected chromosome
Step 2: Validity checking is implemented on two candidates for correct cross operation. If $\sum_{i=\text { start }}^{\text {end }} c_{-} 1(i)$ is equal to $\sum_{i=\text { start }}^{\text {end }} c_{-} 2(i)$, the two candidates are legal; otherwise, go to Step 1.

Step 3: In reality, headways are not constant during the operation, and the average headway cannot be used to prove safety requirements [13]. To avoid train collision, we here consider that the descendants obtained after the cross operation should satisfy the condition that the minimum headway between two adjacent dispatches is not less than $h$, as shown in Figure 8. An unsafe descendant will be discarded and re-generated until it satisfies the safe condition.

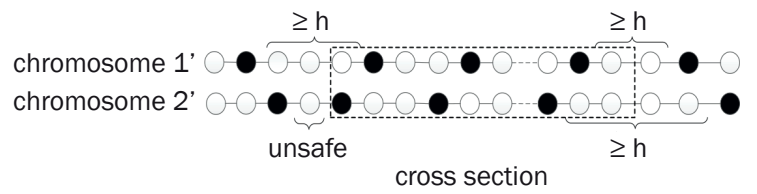

Figure 8 - Safety checking to avoid train collision

Here, we set the constraint that $h$ is larger than the maximal dwell time. In this case, the normal stopping (dwell process) of a train at a station will not affect its following trains unless mis-operations, network failures, or other terrorist activities occur. These special situations are not considered in this study.

Mutation helps the generations to escape from a local optimum by altering one or more gene values in a chromosome from its initial state. The mutation points on a chromosome are selected at random. Then, the selected mutation points on the chromosome mutate from 0 to 1 or from 1 to 0 . After mutation, safety checking is also implemented for the chromosome part of 
the departure times to satisfy the constraint that the minimum headway between two adjacent dispatches is not less than $h$. Otherwise, the mutation will be re-implemented.

\subsection{The fitness function}

When a new population is obtained, we calculate the fitness described by Equation 12 of each individual in order to choose the best individual as the solution of the current generation and provide the basis for the selection in the next iteration. In our study, the stopping criterion for terminating the genetic algorithm is when the algorithm reaches a maximum number of iterations.

Fitness $=\frac{Z_{\max }-Z_{\min }}{Z_{\max }-Z}$

In Equation 12, $Z_{\max }$ and $Z_{\min }$ denote the maximum and minimum of values $Z$, respectively, in the previous generations. In the current generation, we should select the minimal $Z$ and then update $Z_{\min }$. The value ofFitness will increase and approach 1 with the increase of iterations.

\section{CASE STUDY}

We apply our method to the first stage project of line 1 of Nanjing Metro in China, which has 16 stations and is $21.72 \mathrm{~km}$ long, passing across 5 districts, as shown in Figure 9.

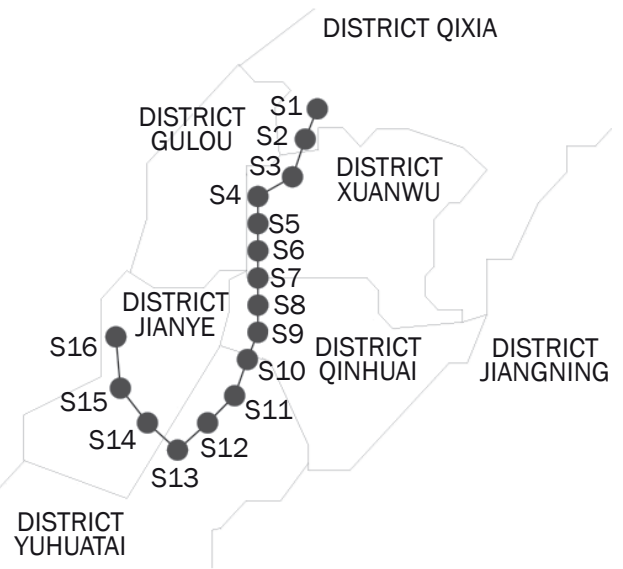

Figure 9 - Case study line

\subsection{The basic information}

We have applied our method to the demand data of the line for many weekdays, and have observed that the passenger waiting time and the number of congested stations can be decreased. Since the demands of different weekdays have a similarly periodic property, here we take a typical weekday data as an example to show the function of our method in detail. Figure 10 shows the demand profile of the line on a typical weekday, and Table 1 shows the parameters used in the case study. In reality, the departure time, the dwell time, and the running time of a train may be influenced by some situations such as irregular and short halt of a train or over-crowding at stations. This will result in a stochastic timetable to some extent. In this paper, for simplicity and convenience of calculations, the dwell time of trains at each station and the running time of trains between two adjacent stations are supposed to be invariable after being optimized. The designed platform capacity of the largest station "Xinjiekou" is 700 persons for and other stations it is 500 persons according to the design manual of the line. At the start station, the operation period of the metro line is from 6:30 a.m. to 9:30 p.m.

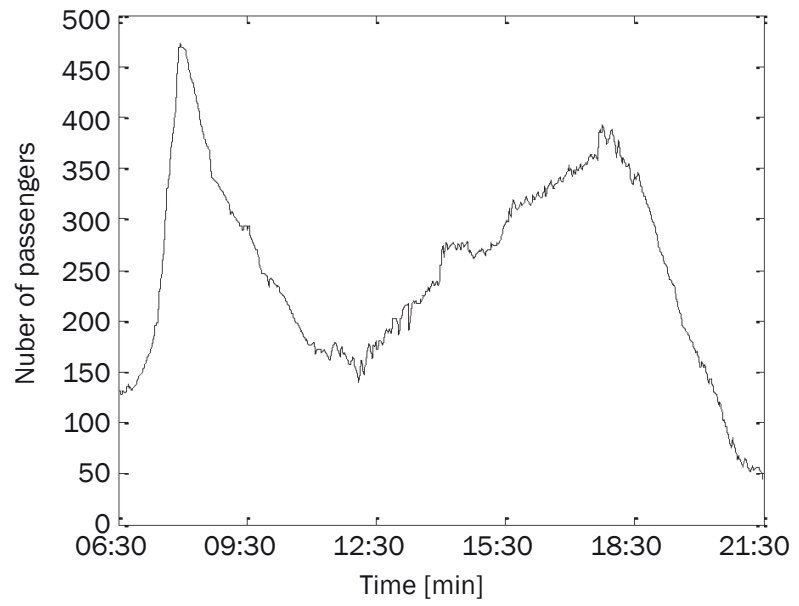

Figure 10 - The demands in terms of the number of passengers on a typical weekday

\subsection{Results}

In this section, we investigate three timetable methods. They are a half-regular timetable method, an irregular timetable method [16] that only focus on the passenger waiting time and the capacity of trains, and our method. For the half-regular timetable method and the irregular timetable method [16], the dwell time and running time are set based on the data provided by Nanjing Metro, as Tables 2 and 3 show. For our method, the dwell time of a station is in the range of [0.2 1.00] minutes, and the running time between

Table 1 - Parameters used in real case

\begin{tabular}{|l|c|c|c|c|c|c|c|c||}
\hline \hline Parameters & Fleet-size & Pull-out time $(T)$ & $h$ & $\alpha$ & $\bar{J}$ & $\begin{array}{c}\text { Population } \\
\text { size }\end{array}$ & $\begin{array}{c}\text { Crossover } \\
\text { probability }\end{array}$ & $\begin{array}{c}\text { Mutation } \\
\text { probability }\end{array}$ \\
\hline \hline Value & 9 & $4 \mathrm{~min}$ & $2 \mathrm{~min}$ & $50 \%$ & $60 \%$ & 40 & 0.95 & 0.1 \\
\hline
\end{tabular}


two adjacent stations is in the range [1.00 3.00] minute, according to the feasibility in real operations. The half-regular timetable method uses the headways as follows: 15 min from $6: 30$ to $7: 30$ and from $20: 30$ to 21:30; 10 min from 7:30 to 20:30. The computer we use has Intel Xeon E3-1230 processor and 16G memory. The GA algorithm is programmed with $\mathrm{C}$ language and executed on the platform of visual studio 6.0. The computation time of the GA algorithm is mainly influenced by the number of iterations, the population size, the modelling time interval, and the chromosome length (number of decision points on a chromosome). According to Figure 6, a chromosome in our case study has 901 points with the interval of 1 minute for the departure time, 272 points with the interval of $0.05 \mathrm{~min}$ ute for the dwell time, and 615 points with the interval of 0.05 minute for the running time. The modelling time interval is 0.2 seconds. Other related parameters are shown in Table 1 . We take about 30 min to obtain the optimal departure time, dwell time, and running time by our method.

In Tables 2 and 3, we show the optimized dwell time and the optimized running time. In Table 4 we show the number of passengers at the congested stations and the corresponding trains. The number of passengers written with a bracket, corresponds to the station number written with a bracket. In Figure 11, we plot the corresponding timetable obtained by our method. We can see that the departure time headways of trains are inhomogeneous, and essentially consistent with the time-dependent demand patterns in Figure 10.
The larger headways correspond to the lower demand, while the high demand pattern is associated with the short headways. In Figure 12, we show the relationship between the fitness and number of iterations for our method. We observe that our method converges with a decreasing speed as the number of iterations increases. The iteration error we use is 0.01 . For clarity, we run and plot 400 iterations here. In Table 5, the statistical results of different timetable methods are presented. As Table 5 shows, our method performs best in terms of passenger travel time. The passenger waiting time of the irregular timetable method [16] is less than that of our method. However, it has more congested events at the stations. The reason for this is that, when the capacity of platforms is ignored, the length of passenger queues at the platforms will be coordinated to be very long when the arriving train has enough capacity. Our method considers the capacity of platforms and trains together, which can avoid this phenomenon and lead to fewer congestion events at the platforms. The main advantage of the half-regular timetable is that the fixed headways can provide better security and convenience for train control. However, it may fail to meet dynamic temporal passenger demand, resulting in long passenger travel time and more congestion events at platforms. Moreover, in real applications, the dwell time extension usually occurs when passengers board or alight trains in over-crowded situations, and the delay of departure time of trains may be induced by irregular and short halt of a train. The dwell time extension and delay of departure time inevitably have

Table 2 - Dwell time of each station [min]

\begin{tabular}{||c|l|c|c|c|l|c|c||}
\hline $\begin{array}{c}\text { Station } \\
\text { number }\end{array}$ & \multicolumn{1}{|c|}{ Station } & $\begin{array}{c}\text { Dwell } \\
\text { time }\end{array}$ & $\begin{array}{c}\text { Optimized } \\
\text { dwell time }\end{array}$ & $\begin{array}{c}\text { Station } \\
\text { number }\end{array}$ & \multicolumn{1}{|c|}{ Station } & $\begin{array}{c}\text { Dwell } \\
\text { time }\end{array}$ & $\begin{array}{c}\text { Optimized } \\
\text { dwell time }\end{array}$ \\
\hline \hline 1 & Maigaoqiao & 0.75 & 0.65 & 9 & Zhangfuyuan & 0.50 & 0.45 \\
\hline 2 & Hongshan Zoo & 0.50 & 0.50 & 10 & Sanshanjie & 0.50 & 0.45 \\
\hline 3 & Nanjing Railway Station & 0.75 & 0.90 & 11 & Zhonghuamen & 0.50 & 0.45 \\
\hline 4 & Xinmofan Road & 0.50 & 0.50 & 12 & Andemen & 0.50 & 0.55 \\
\hline 5 & Xuanwumen & 0.50 & 0.55 & 13 & Xiaohang & 0.50 & 0.40 \\
\hline 6 & Gulou & 0.75 & 0.70 & 14 & Zhongsheng & 0.50 & 0.40 \\
\hline 7 & Zhujiang Road & 0.50 & 0.55 & 15 & Yuantong & 0.50 & 0.55 \\
\hline 8 & Xinjiekou & 0.75 & 0.85 & 16 & Olympic Stadium & 1.00 & 0.80 \\
\hline
\end{tabular}

Table 3 - Running time [min]

\begin{tabular}{||l|c|c|c|c|c|c|c|c|c|c|c|c|c|c|c||}
\hline \hline From & 1 & 2 & 3 & 4 & 5 & 6 & 7 & 8 & 9 & 10 & 11 & 12 & 13 & 14 & 15 \\
\hline To & 2 & 3 & 4 & 5 & 6 & 7 & 8 & 9 & 10 & 11 & 12 & 13 & 14 & 15 & 16 \\
\hline Pre-set & 1.50 & 1.25 & 2.50 & 1.50 & 1.50 & 1.25 & 1.50 & 1.25 & 1.50 & 2.50 & 2.50 & 2.50 & 1.50 & 2.50 & 2.50 \\
\hline Optimized & 1.45 & 1.10 & 2.45 & 1.50 & 1.60 & 1.20 & 1.40 & 1.30 & 1.45 & 2.40 & 2.45 & 2.60 & 1.60 & 2.45 & 2.45 \\
\hline
\end{tabular}


Shen Y, Ren G, Liu Y. Timetable Design for Minimizing Passenger Travel Time and Congestion for a Single Metro Line

Table 4 - The number of waiting passengers at the congested platforms and the corresponding trains for the irregular timetable obtained by our model

\begin{tabular}{|c|c|c|c|c|c|c|c|c|c|c|c|c|c|c|c|}
\hline Station & 6 & 14 & 16 & 67 & 68 & 69 & 70 & 71 & 72 & 73 & 74 & 75 & 76 & 77 & 78 \\
\hline $8(25)$ & 715 & 742 & 815 & 813 & (835) & 848 & 865 & 843 & 845 & (760) & - & (723) & - & - & - \\
\hline $9(24)$ & - & - & - & - & - & 570 & 573 & 610 & 582 & 570 & 586 & (553) & $(560)$ & $(515)$ & - \\
\hline $10(23)$ & - & - & - & - & - & - & - & 542 & - & 580 & 552 & $(560)$ & (565) & $(590)$ & (516) \\
\hline $11(22)$ & - & - & - & - & - & - & - & - & - & 542 & - & 602 & (510) & $(560)$ & (550) \\
\hline $12(21)$ & - & - & - & - & - & - & - & - & - & - & - & - & - & & (530) \\
\hline
\end{tabular}

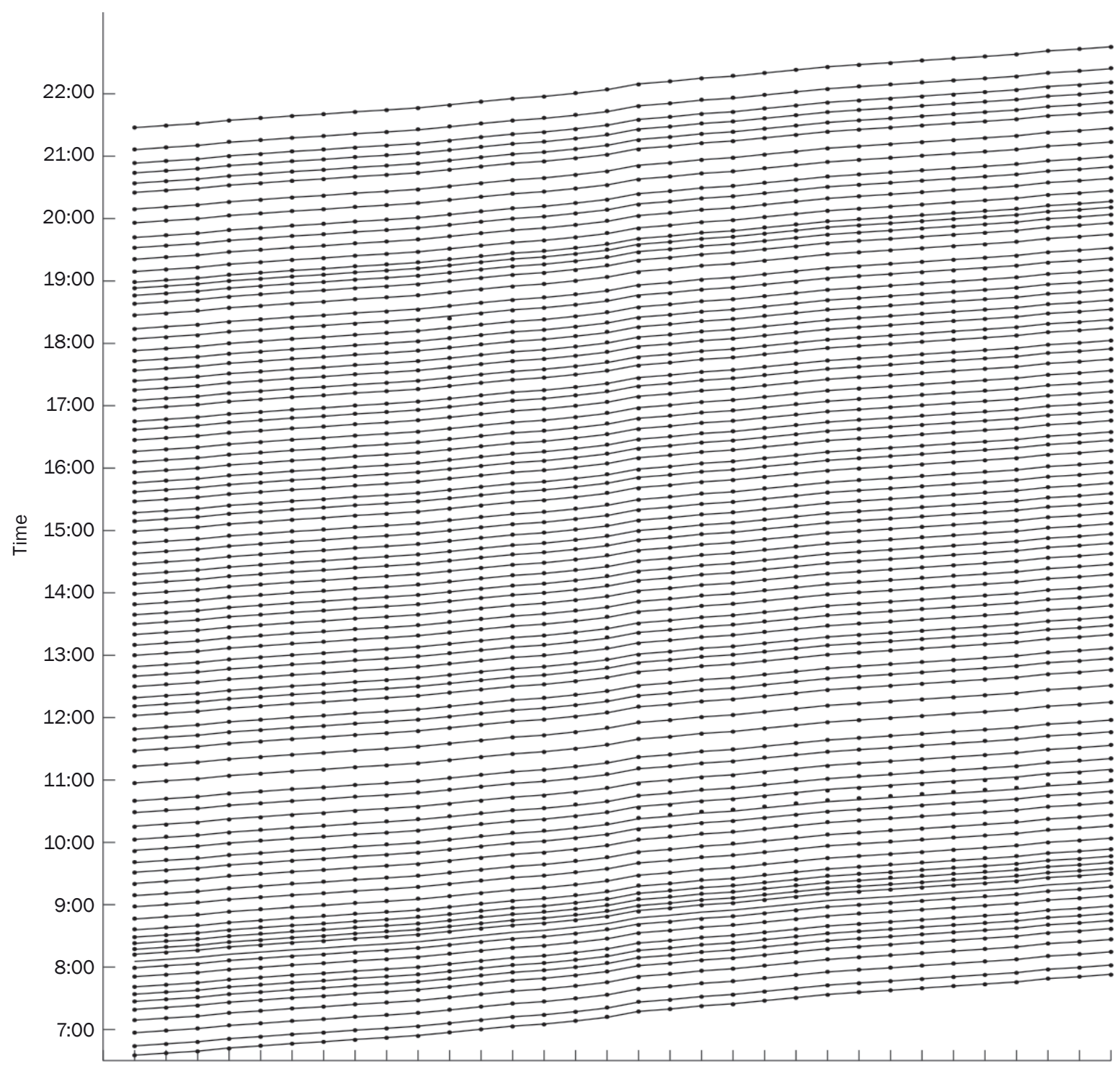

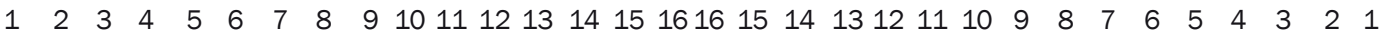

Station

Figure 11 - The optimized timetable scheme for the metro line obtained by our method 
Table 5 - The statistical results of different timetable strategies

\begin{tabular}{||l|c|c|c|c|c||}
\hline \multicolumn{1}{|c|}{ Timetable } & $\begin{array}{c}\text { Number of } \\
\text { dispatches }\end{array}$ & $\begin{array}{c}\text { Average travel time } \\
\text { [min] }\end{array}$ & $\begin{array}{c}\text { Average waiting } \\
\text { time [min] }\end{array}$ & $\begin{array}{c}\text { Average loading } \\
\text { rate }\end{array}$ & $\begin{array}{c}\text { Congested } \\
\text { events }\end{array}$ \\
\hline \hline Half-regular & 86 & 30.52 & 13.92 & $56.31 \%$ & 69 \\
\hline Irregular[16] & 88 & 25.86 & 9.26 & $55.03 \%$ & 52 \\
\hline Our & 88 & 25.03 & 9.38 & $55.03 \%$ & 33 \\
\hline
\end{tabular}

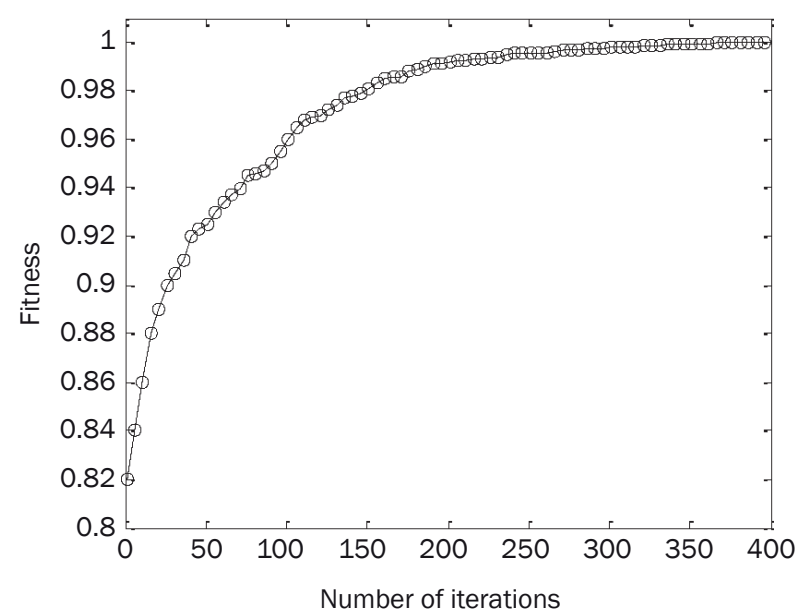

Figure 12 - The relationship between the fitness and the number of iterations for our method

influence on the passenger travel time. To avoid this influence, the original designed operations must be changed. A feasible method is to dynamically adjust the running time of trains. The results obtained by our method can provide a potential solution for dynamically coordinating train resources.

We have also investigated the sensitivity of our method. Table 6 shows the average passenger travel time and computation time for different pull-out times, minimum headways, and population sizes. The

Table 6 - The results of sensitivity analysis of our method number of dispatches is 88. Other parameters are shown in Table 1 and given in Section 4.2. We run each setting 10 times, and average the results. As Table 6 shows, the average travel time decreases with the pullout time and minimum headway. This indicates that low pull-out time and headway can give more adjusting time for realizing higher utilization of train resource and obtaining lower passenger travel time. However, it also brings the difficulty for operations. We can see from Table 6 that the computation time increases with the increase of population size. Larger population size increases the computational burden, and leads to long convergence time. It is noted that the individual diversity is also influenced by very small population size. In the tests, it was found that it is difficult to obtain the global optimal solution when the population size is less than 30 .

It can be inferred that the free-flow state (there are no congested events at platforms) for high demand can be obtained if we increase the number of dispatches of trains. It is meaningful to determine the solution with minimal number of dispatches for obtaining the free-flow state and minimal passenger travel time. In the optimization, the fleet-size may be increased for finding the optimal solution since the number of dispatches is limited under a fixed fleetsize. Table 7 shows the results for different numbers of dispatches with application of our method. The

\begin{tabular}{||c|c|c|c|c||}
\hline $\begin{array}{c}\text { Average travel time } \\
{[\mathrm{min}]}\end{array}$ & $\begin{array}{c}\text { Computation time } \\
{[\mathrm{min}]}\end{array}$ & $\begin{array}{c}\text { Pull-out time } \\
{[\mathrm{min}]}\end{array}$ & $\begin{array}{c}\text { Minimum } \\
\text { headway [min] }\end{array}$ & $\begin{array}{c}\text { Population } \\
\text { size }\end{array}$ \\
\hline \hline 24.32 & 28 & 3.5 & 1.75 & 40 \\
\hline 25.02 & 28 & 4.0 & 2.00 & 40 \\
\hline 25.58 & 27 & 4.5 & 2.25 & 40 \\
\hline 26.25 & 26 & 5.0 & 2.50 & 60 \\
\hline 24.43 & 34 & 3.5 & 1.75 & 60 \\
\hline 25.06 & 35 & 4.0 & 2.00 & 60 \\
\hline 25.50 & 33 & 4.5 & 2.25 & 60 \\
\hline 26.35 & 35 & 5.0 & 2.50 & 80 \\
\hline 24.35 & 38 & 3.5 & 1.75 & 80 \\
\hline 25.05 & 40 & 4.0 & 2.00 & 80 \\
\hline 25.55 & 41 & 4.5 & 2.25 & 80 \\
\hline 26.30 & 40 & 5.0 & 2.50 & \\
\hline
\end{tabular}


related parameters are shown in Table 1 . We concentrate on the transition from congestion to free-flow state. As Table 7 shows, the number of congestion events decreases with the increase of the dispatches. When the number of dispatches increases from 90 to 91, an abrupt decrease of the number of congestion events is observed. The critical point from congestion to free-flow state is meaningful for determining the optimal dispatch of trains that has minimal cost and can avoid congestion. When the number of dispatches arrives at 92 with fleet-size 10, the free-flow state with minimal passenger travel time is obtained. Figure 13 shows the timetable scheme for the 92 dispatches obtained by our model.

Table 7 - The transition from congestion to free-flow state with application of our method

\begin{tabular}{||c|c|c|c|c||}
\hline $\begin{array}{c}\text { Number of } \\
\text { dispatches (fleet-size) }\end{array}$ & $\begin{array}{c}\text { Average waiting time } \\
{[\mathrm{min}]}\end{array}$ & $\begin{array}{c}\text { Average travel time } \\
{[\mathrm{min}]}\end{array}$ & Average loading rate & Congestion events \\
\hline \hline $88(9)$ & 9.38 & 25.03 & $55.03 \%$ & 33 \\
\hline $89(9)$ & 9.21 & 24.74 & $54.41 \%$ & 31 \\
\hline $90(9)$ & 9.13 & 24.63 & $53.81 \%$ & 23 \\
\hline $91(10)$ & 8.84 & 24.24 & $53.22 \%$ & 4 \\
\hline $92(10)$ & 8.42 & 23.92 & $52.64 \%$ & 0 \\
\hline
\end{tabular}

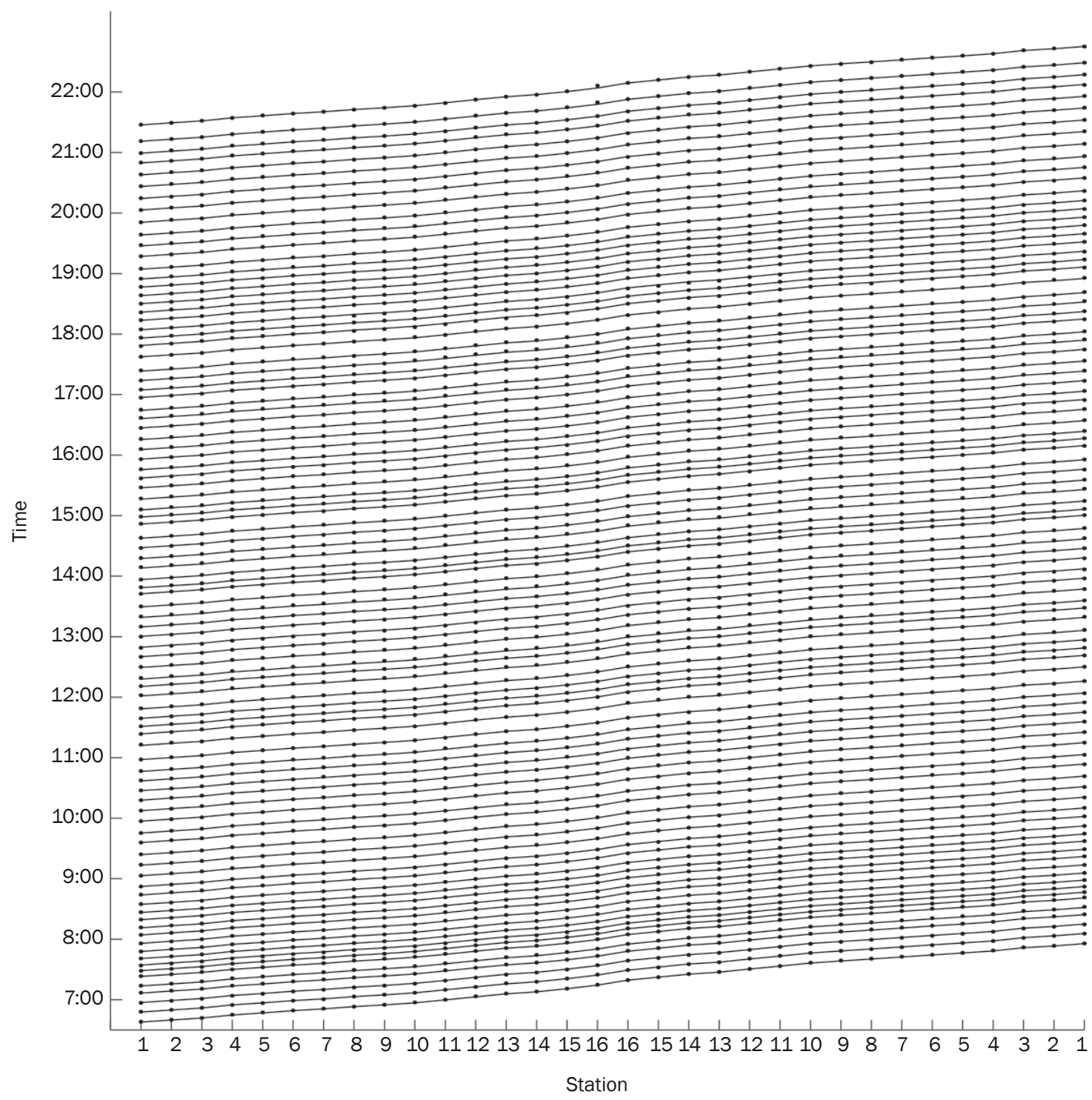

Figure 13 - The optimized timetable scheme for 92 dispatches with free-flow state 


\section{CONCLUSIONS}

This paper presents a timetable optimization model that aims to reduce the passenger travel time and congestion events at platforms for a single metro line under time-dependent demand. The model considers the passenger travel time, the capacity of trains, and the capacity of platforms systematically. A multi-objective function is proposed, and a recursive method incorporated by the genetic algorithm is presented to optimize the model variables. These variables include the departure time of each train at the start station, the dwell time at each station, and the running time between two adjacent stations. The method is applied to a real example and it is compared with two existing methods, a half-regular timetable and an irregular timetable. The results show that our method enables us to obtain a train timetable with minimum passenger travel time and minimum congestion events on platforms. Moreover, by increasing the number of dispatches, the phase transition from congestion state to free-flow state and the optimal timetable with minimal passenger travel time for avoiding congestion on platforms are obtained by our method. The demand-sensitive timetable obtained by our method can be used to coordinate the train resources. A stochastic timetable that derived from the optimal solution with dense dis patch of trains for the peak hours and sparse dispatch of trains for the off-peak hours could be efficient in real applications.

There are two improvements in our further research. (1) Quick optimization algorithms and advanced computation methods should be developed to solve large-scale timetable problems. (2) Our method is based on the known passenger demand data. We will use big data methods to accurately predict the passenger demand, and combine it with our method for practical applications.

\section{ACKNOWLEDGEMENTS}

We acknowledge the Nanjing Metro Corporation for providing the data. This work is also supported by the Natural Science Foundation of China (Grant Nos 51578149 and 51678132), the Key Research and Development Program of Jiangsu Province (Social Development) (Grant No. BE201674), the Chinese Post-doctoral Science Foundation (Grant No. 2016M591747) and the Chinese Scholarship Council (Grant No. 201706855040).

沈毅 1,2 , 任刚 ${ }^{1}$ (通信作者), 刘洋 1,2

1 东南大学交通学院，南京, 210096

2 南京农业大学信息科技学院, 南京, 210095
地铁单线乘客旅行时间与拥塞最小化时刻表设计

\section{摘要}

本文提出了一种在时间变化需求下的乘客旅行时间及 站点拥塞最小化时刻表优化模型。该模型是一种能系统考 虑乘客旅行时间、列车容量及站台容量的混合整数规划模 型。通过提出多目标优化函数及递归优化方法进行模型优 化, 可得出具有最小化乘客旅行时间和最小化站点拥塞事 件数高效时刻表设计方案。不仅如此, 在该模型的基础 上, 通过增加发车次数, 可获得从拥塞到畅通的零界点及 可有效避免站点拥塞的最小成本化时刻表。通过真实数据 评估了该模型的有效性，并和半规则时刻表和非规则时刻 表进行了比较分析。

关键词

地铁; 时刻表优化; 时变需求; 拥塞

\section{REFERENCES}

[1] Alberto C, Matteo F, Paolo T. Modeling and solving the train timetabling problem. Operations Research. 2002;50(5): 851-861. doi:10.1287/opre.50.5.851.362.

[2] Yang X, Li X, Ning B, Tang T. A survey on energy-efficient train operation for urban rail transit. IEEE Transactions on Intelligent Transportation Systems. 2016;17(1): 2-13. doi:10.1109/TITS.2015.2447507.

[3] Newell GF. Dispatching policies for a transportation route. Transport Sci. 1971;5(1): 91-105. Available from: http://dl.acm.org/citation.cfm?id=2691820

[4] Osuna EE, Newell GF. Control strategies for an idealized public transportation system. Transport Sci. 1972;6(1): 52-72. Available from: http://www.jstor. org/stable/25767635

[5] LeBlanc LJ. Transit system network design. Transportation Research Part B: Methodological. 1988;22: 383-390. doi:10.1016/0191-2615(88)90042-2.

[6] Domschke W. Schedule synchronization for public transit networks. OR Spectrum. 1989;11: 17-24. doi:10.1007/BF01721163.

[7] Kwan C, Chang C. Application of evolutionary algorithm on a transportation scheduling problem-the mass rapid transit. Proceedings of the IEEE Congress on Evolutionary Computation. 2005 SEP 02-05; Edinburgh, SCOTLAND: IEEE; 2005.

[8] Liebchen C. The first optimized railway timetable in practice. Transport Sci. 2008;42(4): 420-435. doi:10.1287/trsc.1080.0240.

[9] Wong R, Yuen T, Fung K, Leung JMY. Optimizing timetable synchronization for rail mass transit. Transport Sci. 2008;42(1): 57-69. doi:10.1287/trsc.1070.0200.

[10] Hadas Y, Ceder A. Optimal coordination of public-transit vehicles using operational tactics examined by simulation. Transportation Research Part C: Emerging Technologies. 2010;18: 879-895. doi:10.1016/j. trc.2010.04.002.

[11] Wu JJ, Liu MH, Sun HJ, Li T, Gao Z. Equity-based timetable synchronization optimization in urban subway network. Transportation Research Part C: Emerging Technologies. 2015;51: 1-18. doi:10.1016/j. trc.2014.11.001.

[12] Sun L, Jin JG, Lee DH, Axhausen KW, Erath A. 
Demand-driven timetable design for metro services. Transportation Research Part C. 2014;46: 284-299. doi:10.1016/j.trc.2014.06.003.

[13] Hansen IA, Pachl J. Railway timetabling \& operations. $2^{\text {nd }}$ revised and extended edition. Hamburg, Germany: Eurailpress; 2014.

[14] Albrecht T. Automated timetable design for demand-oriented service on suburban railways. Public Transp. 2009;1: 5-20. doi:10.1007/s12469-008-0003-4.

[15] Barrena E, Cana D, Coelho L, Laporte G. Exact formulations and algorithm for the train scheduling problem with dynamic demand. Computers and $0 p$ erations Research. 2014;44: 66-74. doi:10.1016/j. cor.2013.11.003.

[16] Niu HM, Zhou XS. Optimizing urban rail timetable under time-dependent demand and oversaturated conditions. Transportation Research Part C: Emerging Technologies. 2013;36: 212-230. doi:10.1016/j. trc.2013.08.016.

[17] Niu HM, Zhou XS, Gao RH. Train scheduling for minimizing passenger waiting time with time-dependent demand and skip-stop patterns: Nonlinear integer programming models with linear constraints. Transportation Research Part B: Methodological. 2015;76: $117-$ 135. doi:10.1016/j.trb.2015.03.004.

[18] Xu XY, Liu J, Li HY. Analysis of subway station capacity with the use of queueing theory. Transportation Research Part C: Emerging Technologies. 2014;38(1): 28-43. doi:10.1016/j.trc.2013.10.010.

[19] Transportation Research Board. Transit capacity and quality of service manual. $2^{\text {nd }}$ Edition. TCRP Report 100. Washington, DC; 2003.

[20] Hu QM. Passenger Carrying Capacity Evaluation and Simulation of Rail Transit Station. Ph.D. Dissertation. Beijing, China: Beijing Jiaotong University; 2011.

[21] Chen SK, Liu S. M/G/C/C-based model of passenger evacuation the capacity of stairs and corridors in the metro stations. Journal of the China Railway Society. 2012;34(1): 7-12.

[22] Abril M, Barber F, Ingolotti L. An assessment of railway capacity. Transportation Research Part E: Logistics \& Transportation Review. 2008;44(5): 774-806. doi:10.1016/ j.tre.2007.04.001.

[23] Lam WHK, Cheung CY, Lam CF. A study of crowding effects at the Hong Kong light rail transit stations. Transportation Research Part A: Policy \& Practice. 1999;33(5): 401-415. doi:10.1016/S09658564(98)00050-0.

[24] Zhu YT, Mao BH, Liu L, Li MG. Timetable design for urban rail line with capacity constraints. Discrete Dynamics in Nature and Society. 2015;429219: 11 p. doi:10.1155/2015/429219. 